Research Paper

\title{
Optimizing a 3D Culture System to Study the Interaction between Epithelial Breast Cancer and Its Surrounding Fibroblasts
}

\author{
Liyuan Li and Yi Lu ${ }^{\bowtie}$
}

Department of Pathology and Laboratory Medicine, University of Tennessee Health Science Center, Memphis, Tennessee, USA

Corresponding author: Yi Lu, Ph.D., Department of Pathology and Laboratory Medicine, University of Tennessee Health Science Center, Cancer Research Building, Room 218, 19 South Manassas Street, Memphis, TN 38163, USA. Telephone: (901) 448-5436; Fax: (901) 448-5496; e-mail: ylu@uthsc.edu

( ) Ivyspring International Publisher. This is an open-access article distributed under the terms of the Creative Commons License (http://creativecommons.org/ licenses/by-nc-nd/3.0/). Reproduction is permitted for personal, noncommercial use, provided that the article is in whole, unmodified, and properly cited.

Received: 2011.03.22; Accepted: 2011.08.17; Published: 2011.08.25

\begin{abstract}
Stromal fibroblasts are suggested to be a key determinant in the malignant progression of breast cancer. To find an in vitro culture model that best mimics the in vivo tumor microenvironment so we can study the effects of stromal fibroblasts on breast cancer progression, we evaluated several three-dimensional (3D) co-culture models in order to identify the most suitable culture model for our study. The purpose of our study is to co-culturing malignant mouse breast cancer 4TI cells and murine embryonic fibroblasts (MEF) to form spheroids with matrigel. We found the best culture model for forming the 4TI aggregates/spheroids was, in the absence of fibroblast, by growing 4TI cells in the culture wells precoated with matrigel and in the overlay medium containing $2 \%$ matrigel. We chose this model as our standard 3D culture to co-culture 4TI and MEF cells at different ratios. We found that the amount of MEF in the 4TI/MEF mixture affects the morphology of 4TI/MEF aggregates/spheroids: the higher the ratio of MEF in the mixture, the more ductal structures formed among the aggregates, and the more polarized-like alveolar structures they tended to become. Fibroblasts produced protection for the breast cancer cells in the 3D culture, as aggregates/spheroids formed by breast-cancer cells alone were more sensitive to cytotoxic chemo-agents than aggregates formed by the breast-cancer/fibroblast mixture. These results indicate that the selection of a suitable 3D culture model for a particular research focus may be critical to collecting clinically relevant information about tumor progression that involves interplay between different cell types. This 3D co-culture model demonstrated that tumor-surrounding fibroblasts play important roles in distributing and connecting epithelial breast cancer cells in a tumor microenvironment, as well as providing protection for breast cancer cells from chemo-agent killing.
\end{abstract}

Key words: 3D co-culture model, breast cancer cells

\section{INTRODUCTION}

Breast cancer (BCa) is the most frequently diagnosed cancer and the second leading cause of cancer deaths in American women today. It is estimated that there were 207,090 new cases of BCa and 39,840 BCa deaths in American women in 2010 (1). The evaluation of newly developed anti-cancer drugs are usually performed initially in the traditional two-dimensional
(2D) culture; however, most drugs that show promise in the 2D culture are rarely effective in vivo in animals or clinical trials, partially because the clinically relevant breast tumors do not exist as a monolayer, stand-alone entity in vivo. Thus, to better study BCa malignant progression that involves interplay between different cell types and to accurately evaluate 
the therapeutic efficacy of anticancer drugs, a better in vitro co-culture model that more closely resembles the in vivo $\mathrm{BCa}$ growth environment should be identified and used.

Cancer cells in vivo live in a complex microenvironment consisting of non-cancer cells and tumor stroma, including basement membrane, extracellular matrix (ECM), blood vasculature, inflammatory cells and fibroblasts, all are shown to contribute to cancer development (2-4). The importance of stroma for mammary carcinogenesis has been recently discovered as well as how tumor stroma contributes to the malignant progression of epithelial tumors (5). Studies show that mammary gland stroma is able to either promote or prevent carcinogenesis in mammary epithelial cells: carcinogenic stimulation of mammary gland stroma promots tumorigenesis in normal mammary epithelial cells $(6,7)$, while normal mammary gland stroma induces normal ductal growth of grafted epithelial tumor cells and prevents their neoplastic development (8). Fibroblast was the major component in the stroma and plays a predominant role in tumor progression $(9,10)$. As a principal constituent of the stroma, fibroblast is responsible for the production of growth factors, extracellular matrix, angiogenic and immunological chemokines that promote tumor growth $(9,10)$. Stromal fibroblasts are believed to adapt and continuously co-evolve with tumor epithelial cells' transformation and development (13). Therefore, fibroblasts are a key determinant in the tumor malignant progression and can be used to target for cancer therapies. The study of breast tumors along with the associated stroma fibroblast as an entity would provide significant insights to tumor progression that involves interplay between different cell types and to clinically relevant evaluations of therapeutic drugs.

While tumor tissues or animal models have been frequently used to study the interplay between stromal fibroblast and tumor cells, these approaches are sometimes complex and intractable, presenting challenges for interpreting discrepancies (14) in addition to other difficulties and costs dealing with tissues and animals. On the other hand, traditional 2D cell culture, the widely used standard monolayer culture for mammary tumor cells, provides few physiologic resemblance of a tumor growth environment in vivo attributed from morphology, cell-cell and cell-matrix interactions. To fill in the gap between the monolayer cell culture and whole animal, the 3D in vitro culture has emerged as a third approach that mimics the in vivo cell growth environment. The goal of the $3 \mathrm{D}$ culture is to permit researchers to investigate the cellular signaling mechanisms and effects of anti-tumor agents in a closely resembling in vivo situation $(14,15)$ while taking advantage of in vitro culture's simplicity and low cost (as opposed to the tissues/animals' complexity, intractability and high cost).

As the objectives for developing 3D culture systems may vary widely, ranging from engineering tissues for clinical deliveries through to the development of models for drug screening (16), the selection of a suitable 3D culture becomes important for studying the relevant research aims. The goal of this study was to compare several routine 3D culture models to select the best one to suit our purpose to mimic and study the interaction between epithelial $\mathrm{BCa}$ and stromal fibroblasts in vivo in a tumor growth microenvironment. We also examined the effects of the ratio of the co-cultured 4T1 BCa cells and MEF fibroblasts on the morphology of the formed cell spheroids and protection shield from fibroblast to the spheroids against chemo-agent.

\section{MATERIALS AND METHODS}

\section{Cell lines and culture conditions}

Mouse mammary carcinoma cell line 4T1 (gift of Dr. C. Liu of Scripps) was grown in RPM1-1640 medium (Invitrogen, Grand Island, NY) containing 10\% fetal bovine serum (FBS) (Hyclone Laboratories, Logan, UT). Murine embryonic fibroblasts (MEF) were grown in Dulbecco's modified Eagle medium (DMEM) with high glucose and $25 \mathrm{mM}$ HEPES (Gibco BRL, Carlsbad, CA). Mouse mammary tumor epithelial cell line PyT (gift from Dr. T. Seagroves of UTHSC) was grown in DMEM/F12 (1:1) with FBS. All cell lines were grown in medium containing 100 units/ $\mathrm{ml}$ penicillin, and $100 \mu \mathrm{g} / \mathrm{ml}$ streptomycin. The cell cultures were incubated at $37^{\circ} \mathrm{C}$ in $5 \% \mathrm{CO}_{2}$.

\section{Generation of 4TI expressing GFP}

The construction of the Tet-on green fluorescent protein (GFP) lentiviral system was described previously (17). 4T1 cells stably infected with lentiviral system expressing Tet-on GFP were generated (4T1/GFP). For induction of GFP transgene expression, $1 \mu \mathrm{g} / \mathrm{ml}$ doxycyline (Dox) (Clontech) was added in the medium for induction of GFP throughout all the experiments.

\section{Three-dimensional (3D) BCa culture system}

Various 3D culture models were examined to determine the suitable $\mathrm{BCa}$ culture system for our study: (i) BCa cells growing in culture wells precoated with matrigel (BD Biosciences, Bedford, MA) and in regular medium (3D-base), (ii) $\mathrm{BCa}$ cells growing in culture wells without base matrigel and in medium 
containing $2 \%$ matrigel (3D-no base and embedded), or (iii) BCa cells growing in culture wells precoated with matrigel and in medium containing $2 \%$ matrigel (3D-base and embedded). The last system (3D-base and embedded) was chosen in our studies and is herein referred as the "standard 3D culture" system throughout.

4T1 BCa cells were seeded into 96-well plates at a density of $1 \times 10^{4}$ cells/well. In the standard 2D culture model: cells suspended in culture medium were directly plated into 96-well culture plate. In the 3D-base model, the 96-well plates were precoated with matrigel as basement membrane by adding $40 \mu \mathrm{l}$ of matrigel to each well followed by incubating at $37^{\circ} \mathrm{C}$ for $30 \mathrm{~min}$ to let the matrigel solidify, then cells were plated onto the gel in the regular medium. In the 3D-no base and embedded model, cells were suspended in medium containing $2 \%$ matrigel and then seeded directly onto the uncoated 96-well culture plates. In the standard 3D model (i.e., the 3D-base and embedded model), the 96-well plates were precoated with matrigel as described above, then the cells suspended in medium containing $2 \%$ matrigel were seeded onto the matrigel-precoated wells. All microscopical images were captured using Nikon TE 300 (Nikon, Japan), and a software of NIS-Elements BR 3.0 (Nikon, Japan).

\section{D co-culture system of epithelial BCa cells and fibroblasts}

MEF cells were co-cultured with 4T1 cells in the standard 3D culture (i.e., 3D-base and embedded system). The 96-well plates were precoated with matrigel as described above. A serial cell mixtures with different ratio of $4 \mathrm{~T} 1$ versus MEF (4T1:MEF as 1:0, 6:1, $3: 1,1: 1,1: 3,1: 6)$ were prepared in medium containing $2 \%$ matrigel. The mixtures of $4 \mathrm{~T} 1$ and MEF cells (at different ratio but with a fixed total cell number as 10,000 cells/well) were directly seeded onto the matrigel-precoated 96-well plate. The cells were incubated at $37^{\circ} \mathrm{C}$ for up to 10 days to allow the aggregates/spheroids to form, the medium was changed every 4 days. Starting day 5 , the cells in co-culture system were periodically taken fluorescent microscopic images to analyze the morphology. The wells without cells but containing medium were used as negative control. All assays were performed at least three times and each time was tested in triplicate.

\section{Drug treatment of $\mathrm{BCa}$ cell spheroids in standard 3D culture}

Epithelial BCa PyT cells were mixed with MEF (PyT:MEF ratio as 1:3) or without MEF cells, a total of $1 \times 10^{4}$ cells in medium containing $2 \%$ matrigel were seeded onto the 96-well precoated with matrigel (the standard 3D culture). The cells were incubated at $37^{\circ} \mathrm{C}$ for 5-10 days to allow the aggregates/spheroids to form, the medium were changed every 4 days. To examine the sensitivity of spheroids to the chemo-agent YC-1 (Alexis Biochemicals Corp., San Diego, CA), the old medium in the standard 3D culture was carefully removed and replaced with fresh medium containing a serial concentration of YC-1 at final concentration as $0.1,1,10$ and $100 \mu \mathrm{M}$. Starting day 4 after the drug treatment, the size of spheroids were measured periodically up to 3 weeks with medium change every 4 days.

\section{RESULTS}

\section{Establishment of 4TI cells expressing GFP}

To help distinguish epithelial 4T1 BCa cells from MEF fibroblasts or matrigel basement membrane in the 3D culture, we used the 4T1 line expressing GFP under an inducible Tet-on promoter (4T1/GFP) for the study. The inducible 4T1/GFP line was generated by infecting 4T1 cells with Lenti-Tet-on GFP system (17). The inducible GFP expression was used to identify $4 \mathrm{~T} 1$ cells under the fluorescent microscope. The 1 $\mu \mathrm{g} / \mathrm{ml}$ Dox inducer per se does not affect the morphology of $4 \mathrm{~T} 1$ cells in either 2D or 3D culture (data not shown).

\section{Effect of matrigel on BCa cell morphology in a 3D culture system}

To establish a culture system to more closely mimic the mammary cell microenvironment in vivo than the conventional 2D monolayer culture, we compared several different 3D culture systems in order to determine which $3 \mathrm{D}$ culture system is most suitable to our study and simulates the in vivo relevant morphological phenotype. First, we examined effects of basement membrane (matrigel) on BCa cell morphology in three commonly used 3D culture models, which are BCa cells growing on basement membrane (matrigel), or in medium containing matrigel, or both (Fig. 1). Correspondingly, we let 4T1 cells grow in culture wells precoated with matrigel (3D-base, Fig. 1B) in regular medium, or without base matrigel but in medium containing $2 \%$ matrigel (3D-no base and embedded, Fig. 1C), or with matrigel in both precoated wells and medium (3D-base and embedded, or so-called standard 3D culture in this paper, Fig. 1D). After 5 days in culture, the morphogenesis of $4 \mathrm{~T} 1$ cells was evaluated in these $3 \mathrm{D}$ culture systems along with the traditional 2D culture. $4 \mathrm{~T} 1$ cells in 2D culture proliferated to form a monolayer as expected (Fig. 1a'). Neither 4 T1 cells in medium containing $2 \%$ matrigel 
alone without base-coated matrigel (Fig. 1c') nor 4T1 cells growing on precoated matrigel alone without matrigel in medium (Fig. $1 b^{\prime}$ ) formed the cell aggregates/spheroids; only the culture condition where matrigel was present both in the precoating wells and in the medium was able to promote $4 \mathrm{~T} 1$ cells to form cell aggregates/spheroids (Fig. 1d'), a more realistic, in vivo-like phenotype of BCa cells $(18,19)$. These results suggest that the support of matrigel in the microenvironment from both the base and surrounding is important for $\mathrm{BCa}$ cells to form aggregates/spheroids, a clinically relevant phenotype in the $3 \mathrm{D}$ culture.

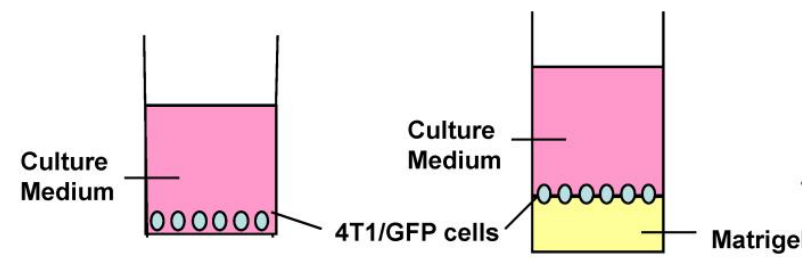

(A) Standard 2D

(B) 3D-base
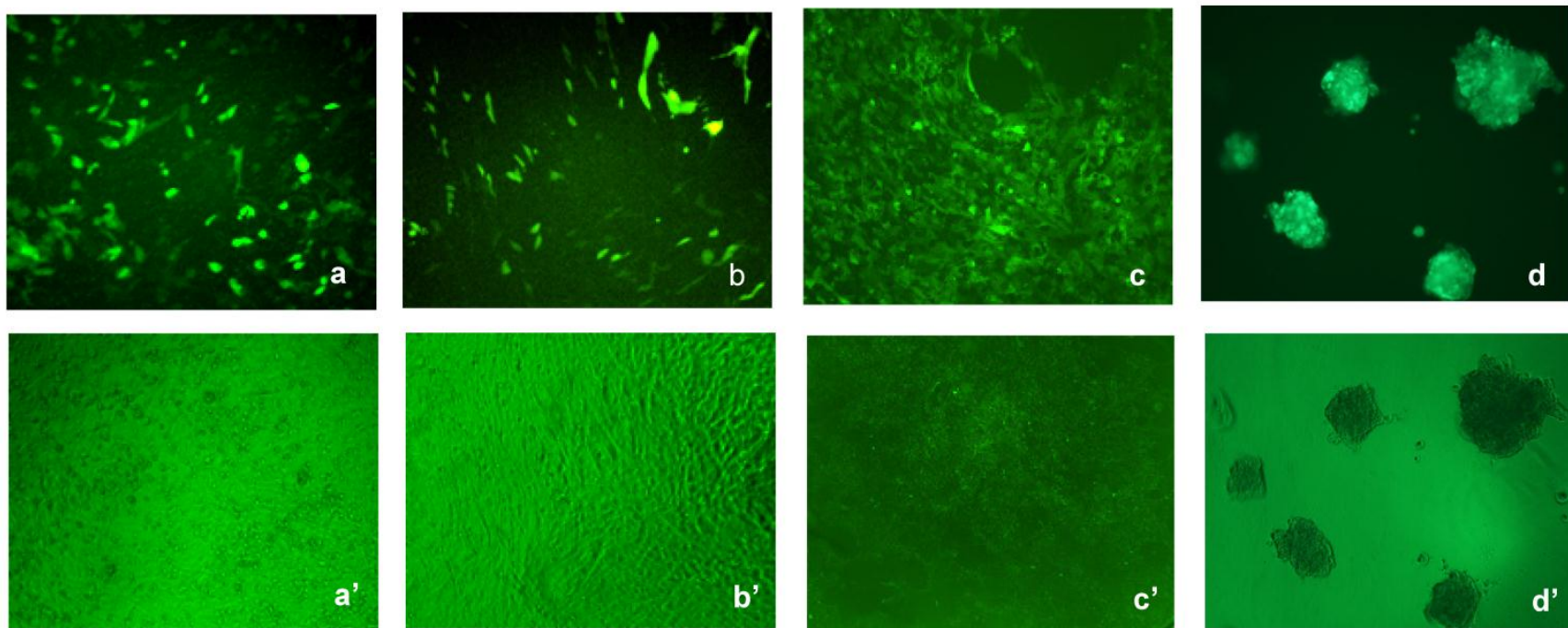

The multi-cell spherical colonies formed in the 3D-base and embedded culture (i.e., the standard 3D culture, Fig. 1D) appeared to be embedded at different layers in the medium containing $2 \%$ matrigel, as they presented a hierarchy suspended status among the matrigel-containing medium instead of locating at the same layer (Fig. 2). These embedded 4T1 spheroids may provide a spatially and physiologically more relevant breast tumor phenotype for analyzing $\mathrm{BCa}$ morphogenesis and gene function in vivo. We therefore chose this standard 3D culture system (Fig. 1D) for the further studies on co-culturing of epithelial $\mathrm{BCa}$ cells and fibroblasts.

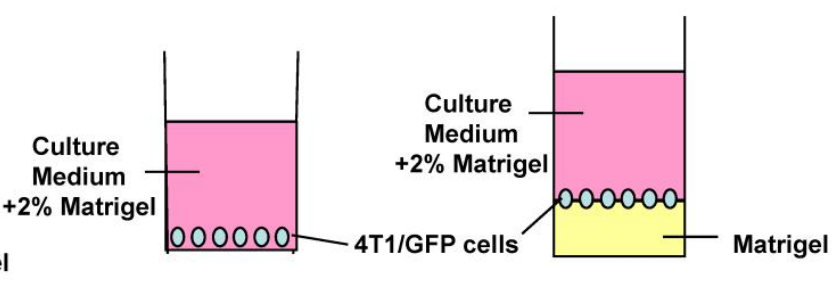

(C) 3D-no base \& embedded

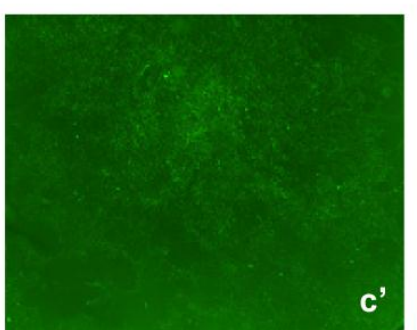

(D) 3D-base \& embedded (Standard 3D)

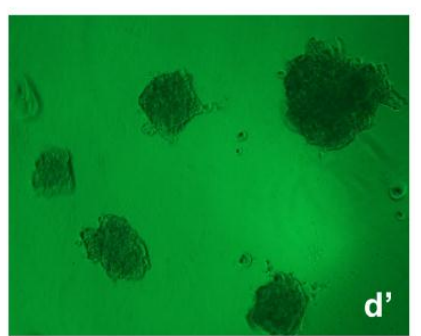

Figure I. Design of various 3D culture systems and their corresponding morphologies. BCa cell line 4TI stably expressing GFP was placed in either standard 2D culture (A) or various 3D cultures (B-D) differing in incorporation of matrigel component in the medium or base of the wells. 4TI/Tet-on GFP cells were seeded in 96 -well plates at a density of $1 \times 10^{4}$ cells/per well in medium containing $I \mu \mathrm{g} / \mathrm{ml}$ Dox. Shown are the morphologies after 5 days in the culture after cell plating. (A) Standard 2D culture: cells suspended in culture medium were directly plated into 96 -well culture plate. (B) 3D-base culture: a thin layer of matrigel were precoated in 96-well plate as basement membrane following by plating cells onto it in the regular medium. (C) 3D-no base and embedded culture: cells were suspended in medium containing $2 \%$ matrigel and then seeded directly onto the uncoated 96-well culture plates. (D) Standard 3D culture: cells were suspended in medium containing $2 \%$ matrigel and seeded onto 96 -well plate precoated with matrigel as basement membrane. Top row (A-D), schematic display of the 2D and 3D culture models. Middle row (a-d), fluorescence microscopy images. Bottom row (a'-d'), light contrast micrograph images. Original magnification: $x 10$. 

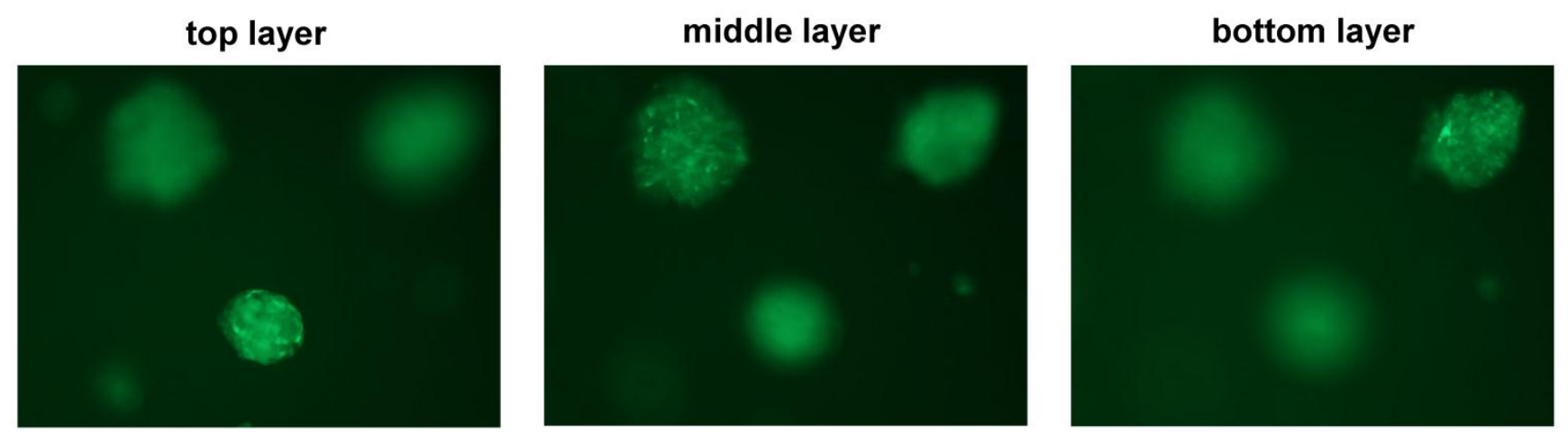

Figure 2. Hierarchy presence of embedded structures of 4TI spheroids in standard 3D culture. Confocal microscopical photos taken at different depth (focused at the top, middle, and bottom layer of the same field, respectively) of some representative 4TI/GFP aggregates/spheroids growing in the standard 3D culture.

\section{Effects of fibroblasts on the BCa cells in a co-culture 3D system}

In order to see the effect of fibroblasts on epithelial BCa cells, different ratios of $4 \mathrm{~T} 1$ and MEF cells were mixed and co-cultured in the standard 3D model along with 4T1 alone 3D culture. By comparison, the co-cultured 4T1/MEF mixture tended to form better and bigger spheroids than those formed by $4 \mathrm{~T} 1$ cells alone (compare Fig. 3B, 3C with Fig. 3A). The higher the ratio of fibroblasts (MEF) in the mixture, the more ductal/tubular network formed among the aggregates (compare among Fig. 3F, Fig. 3D, Fig. 3B), indicating that fibroblasts may facilitate the ductal/tubular network among the 4T1 cells in 3D. Moreover, the amount of surrounding fibroblasts also affected the morphology of the 4T1/MEF spheroids: as shown at a higher magnification in Fig. 4, the higher amount of fibroblasts in the mixture, the more polarized alveolar structures they tended to become. The spheroids of $1: 1$ ratio (50\% MEF) of $4 \mathrm{~T} 1$ :MEF had a more "polarized-like" alveolar structures than those of $3: 1(33.3 \% \mathrm{MEF})$ and $6: 1(16.6 \% \mathrm{MEF})$ ratio of 4T1:MEF (compare Fig. 4C with Fig. 4A and 4B). It appeared that at 1:1 ratio, the $4 \mathrm{~T} 1 / \mathrm{MEF}$ spheroids started to form the incipient alveolar/glandular structures in the 3D co-culture (Fig. 4C). Noticeably, although increased MEF ratio promoted alveoli towards polarized state (compare Fig. 4A, 4B with 4C), the complete alveolar/ductal mammary gland structures were not formed/polarized (Fig. 4C). The longer culture time (up to 3 weeks) did not change the general pattern of alveoli or ductal network (not shown). Together, these results demonstrated that tumor cell-associated fibroblasts play important roles in dis- tributing and connecting BCa cells in a tumor microenvironment.

\section{Fibroblasts protect 4TI cells in the 4TI/MEF mixture from chemo-agent's cytotoxic attack}

3D culture appears to be a better model to predict efficacy of an anticancer drug than 2D culture. Cancer cells including BCa cells are usually more sensitive to cytotoxic chemo-agents in the $2 \mathrm{D}$ culture than in the 3D culture. In our study, chemo-agent YC-1, an anti-hypoxia inducible factor and anticancer drug (20), killed BCa PyT and 4T1 cells more effectively in the $2 \mathrm{D}$ culture than in the $3 \mathrm{D}$ culture. For example, $10 \mu \mathrm{M}$ YC-1 killed $92 \%$ of PyT cells in the 2D culture while the same concentration of the drug only affected 34\% PyT cells in the 3D culture (our unpublished results). The discrepancy of drug sensitivity in the 2D and 3D culture may partially explain why so many drugs developed based on 2D-culture screen do not work effectively in the clinical situation.

To examine the differential sensitivity to chemo-agent in the 3D culture by $\mathrm{BCa}$ cells alone and by BCa cells mixed with fibroblasts, PyT BCa cell line (21) was chosen for the study because PyT alone (in the absence of fibroblasts) formed better spheroids/aggregates than $4 \mathrm{~T} 1$ alone. As shown in Fig. 5, PyT-alone spheroid is more sensitive to chemo-agent YC-1 than PyT/MEF (1:3) mixed spheroid. When the PyT-alone spheroids shrank and disappeared at 10 $\mu \mathrm{M}$ and higher concentration of YC-1 after 21 days treatment, the PyT/MEF mixed spheroids maintained the size in both $10 \mu \mathrm{M}$ and $100 \mu \mathrm{M}$ YC-1 (Fig. 5). By day 21 after $100 \mu \mathrm{M}$ YC-1 treatment, the size of PyT-alone spheroid had an average of $55.5 \%$ reduction compared to merely $13.33 \%$ reduction in 
PyT/MEF mixed spheroid (Fig. 5). These results suggest fibroblasts in the mixed $\mathrm{BCa} / \mathrm{MEF}$ spheroid provided a shield protection for the spheroids from the cytotoxic attack of chemo-agent. These results imply that a more sophisticated drug evaluation system needs to be applied in addition to the traditional 2D culture for screening/measuring the efficacy of anticancer agents.
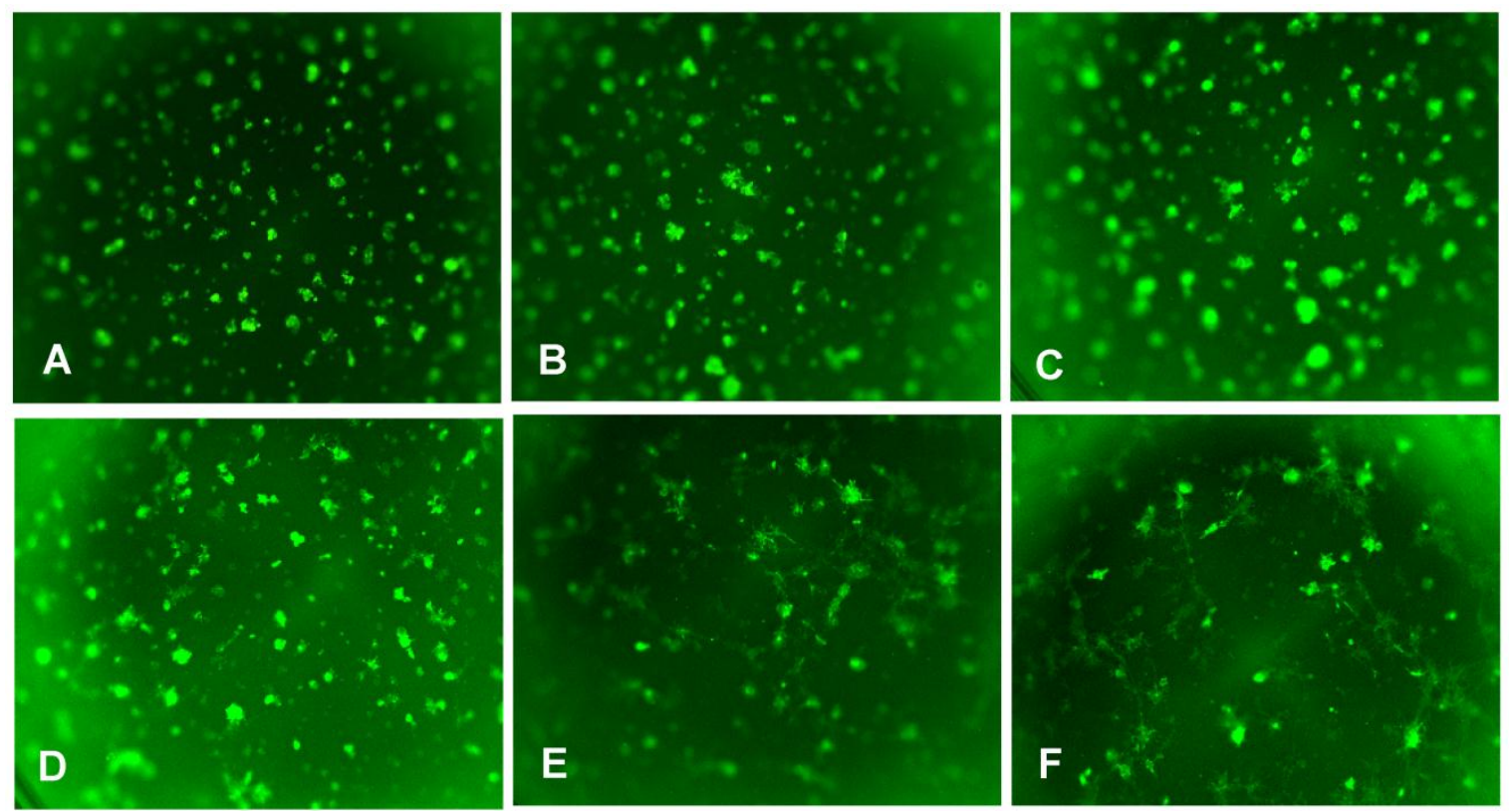

Figure 3. Fibroblasts facilitate the tubular network formation among the epithelial BCa cells. 4TI and MEF were co-cultured at different ratio, and a total $1 \times 10^{4}$ cells/per well were seeded in a 96 -well plate as a standard 3D culture. The cells were incubated at $37^{\circ} \mathrm{C}$ for $5-10$ days to allow the aggregates/spheroids to form, the medium were changed every 4 days. Shown are the photos of (A) 4TI alone, or 4TI/MEF at the ratio of (B) 6:I, (C) 3:I, (D) I:I, (E) I:3, and (F) I:6. Original magnification: $\times 2$.

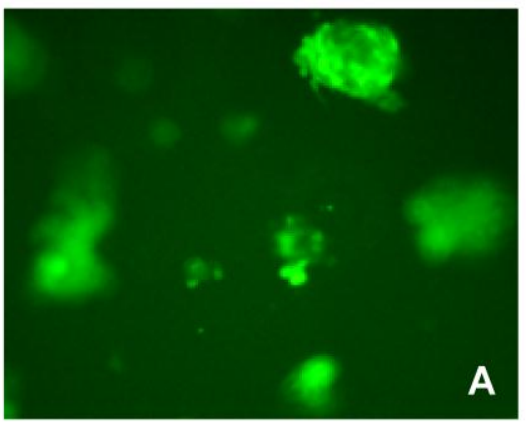

4T1/MEF (6:1)

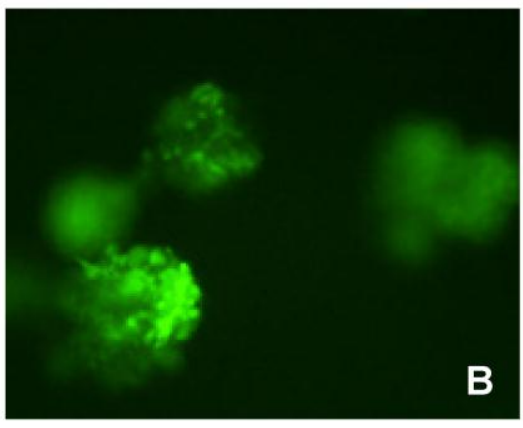

4T1/MEF (3:1)

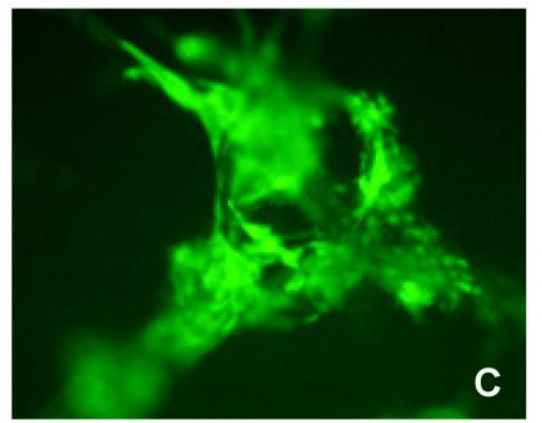

4T1/MEF (1:1)

Figure 4. Morphological changes of alveolar mixture by ratio of surrounding fibroblasts. 4TI/GFP and MEF mixture (a total Ix $10^{4}$ cells/per well) were seeded in 96-well plates in the presence of I ug/ml Dox as a standard 3D culture. The cells were incubated at $37^{\circ} \mathrm{C}$ for $5-10$ days to allow the aggregates/spheroids to form, the medium were changed every 4 days. Shown are the representative images of co-cultured mixture at the ratio (4TI/MEF) of (A) I:I, (B) 3:I, and (C) 6:I, Original magnification: $x 10$. 


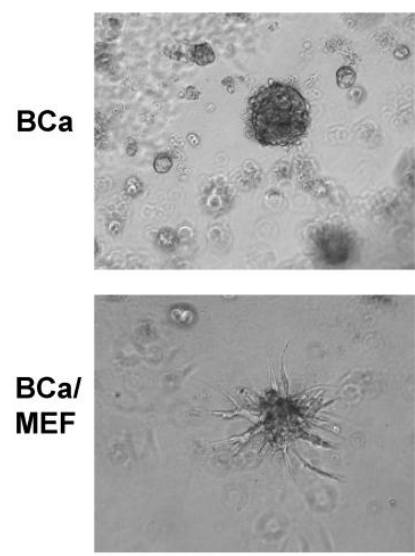

Untreated
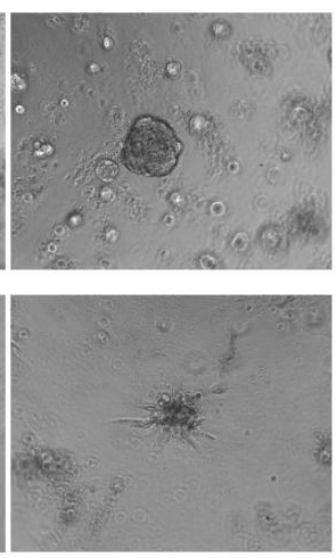

$0.1 \mu \mathrm{M}$ YC-1
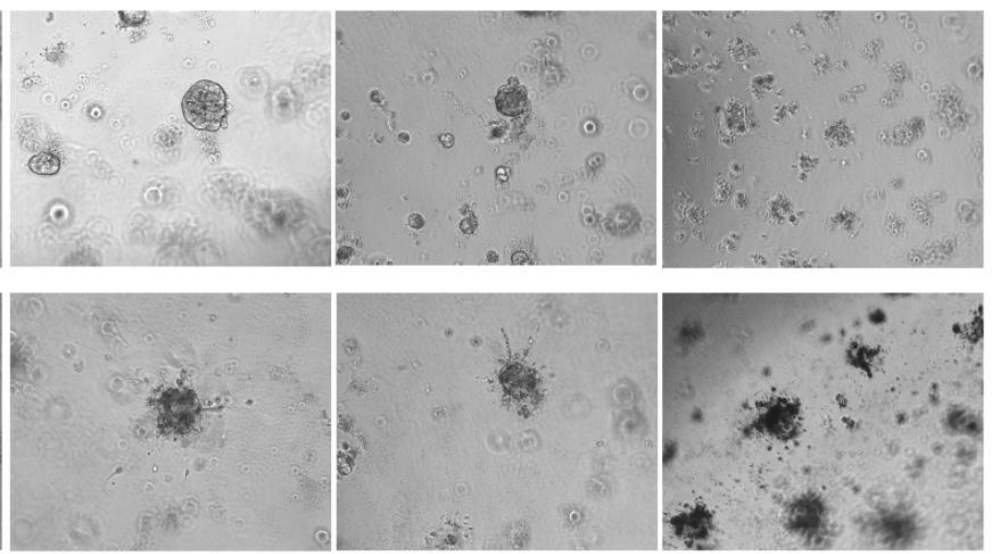

$1 \mu \mathrm{M}$ YC-1

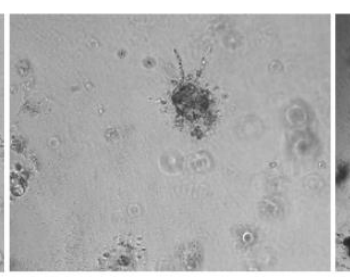

$10 \mu \mathrm{M} Y \mathrm{C}-1$

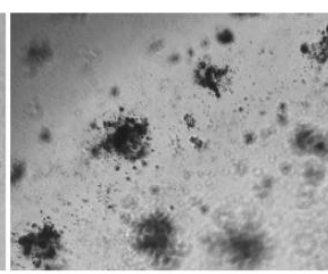

$100 \mu \mathrm{M}$ YC-1

Figure 5. Drug sensitivity of cell spheroids of $\mathrm{BCa}$ alone and $\mathrm{BCa}$ /fibroblast mixture. Epithelial $\mathrm{BCa}$ PyT cells were mixed with or without MEF cells, a total of $1 \times 10^{4}$ cells in medium containing $2 \%$ matrigel were seeded onto the 96 -well precoated with matrigel to form the spheroids (a standard $3 \mathrm{D}$ culture). The cells were incubated at $37^{\circ} \mathrm{C}$ for $5-10$ days to allow the formation of aggregates/spheroids. After carefully removing the old medium, the spheroids were incubated with medium containing a serial concentration of YC-I. Shown are the images of spheroids of BCa PyT cells alone (upper row) and spheroids of PyT/MEF mixture (I:3, lower row) on $2 \mathrm{I}$ days after drug treatment without or with $0.1, \mathrm{I}, \mathrm{I}$, and $100 \mu \mathrm{M}$ YC-I.

\section{DISCUSSION}

Compared to the traditional 2D culture, known as the simplest model without most in vivo characteristics, the 3D culture has been increasingly accepted as a more physiologically relevant and predictive model. Emerging evidences have shown that tumor growth is not just determined by malignant cancer cells themselves, but also by the tumor microenvironment $(10,22)$. Correspondingly, the co-culture 3D system should provide a better in vitro culture model for studying tumor progression that involves interplay between different cell types and evaluation of efficacy of anticancer drugs. For example, our results showed YC-1, an anti-cancer chemo-agent that induces cell cycle arrest and apoptosis via HIF-1 $\alpha$ suppression (24), killed BCa cells much less effectively in the 3D culture than in the 2D culture (Fig. 5), consistent with the fact that many drugs developed based on the $2 \mathrm{D}$ culture do not work effectively in the clinical situation. These results suggest that in vitro drug evaluation in 3D culture may provide a better clinical relevant prediction than the traditional 2D culture.

The commonly used 3D culture models including growing tumor cells on matrigel/basement membrane components alone $(18,23)$, in medium containing matrigel/basement membrane components alone (11), or both (12). With the goal to co-culture $\mathrm{BCa} 4 \mathrm{~T} 1$ and fibroblast MEF in order to simulate the in vivo morphology/phenotype of breast tumors, we compared and assessed these different in vitro $3 \mathrm{D}$ models and selected the best suitable $3 \mathrm{D}$ culture--where 4T1 and MEF were embedded in medium with $2 \%$ matrigel and plated onto the wells precoated with matrigel. By analyzing the morphology and drug sensitivity of the co-cultured $\mathrm{BCa} / \mathrm{MEF}$ in this 3D culture model and optimizing with different ratios of $\mathrm{BCa}$ cells and fibroblasts, we found that this $3 \mathrm{D}$ co-culture system resembles the morphological phenotype of breast tumor as the multi-celled spheroids. At the ratio (BCa:MEF) of $50 \%$ or higher MEF amount, the mixed spheroids mimic mammary gland physiological architecture and tumor microenvironment. Thus, this 3D co-culture model is suitable for studying the interaction between epithelial BCa cells and surrounding fibroblasts. For example, we are going to employ this 3D culture model to investigate the paracrine effects of growth factors (including VEGF) stimulated by HIF-1 $\alpha$ from stromal fibroblasts to breast tumor malignant progression. Such a study can be approached by growing MEF/H1F-1 $\alpha$ wild-type or MEF/H1F-1 $\alpha$ knockout cell line as fibroblasts, respectively, in the standard 3D model with co-cultured epithelial BCa cells.

4T1 cells can form aggregate/spheroid (a more physiologically relevant phenotype) in the 3D culture but not in 2D culture (Fig. 1), suggesting the support of matrigel or fibroblasts in the microenvironment is important for forming the clinically relevant phenotype of BCa cells. Particularly, the basement membrane precoating (such as matrigel) is critical for this 
3D culture to promote BCa cells to form a spheroid morphology. The comparison of matrigel-precoating only 3D culture (3D-base, Fig. 1B) with the standard 3D culture (3D-base and embedded, Fig. 1D), suggests that ECM deposited around and between cells in 3D culture is also vital for BCa cells to recapitulate in vivo features, besides bottom-coated basement membrane. The incorporation of fibroblasts promoted spheroids to form "polarized-like" alveolar and ductal structure (Fig. 4C), reminiscent of structures found in vivo. The morphological phenotype of co-cultured 4T1/MEF spheroids can be altered depending on the ratio of 4T1 to MEF: the ductal networks were formed exclusively in the presence of higher amount of MEF (above 50\% MEF in the ratio) in co-culture. The more MEF, the more ductal/tubular networks that were formed (Fig. $3)$.

The previous study by Krause et al. (11) using a $3 \mathrm{D}$ co-culture model, where normal mammary epithelial cells MCF-10A and normal human fibroblasts embedded in medium containing collagen I or mixed matrigel-collagen, showed better polarized mammary gland structure than our model. However, we could not form the sustained 4T1 spheroids in the similar model (3D-base/embedded, Fig. 1C) by embedding $4 \mathrm{~T} 1$ cells in the medium containing $2 \%$ matrigel alone without precoating matrigel (Fig. 1C). We do not know whether it is due to the difference between normal and malignant breast epithelial cells or due to the particular components of basement membrane used in these two studies. The mammary tumor cells, which exhibit significant deviation from normal epithelial cells, may undermine the formation of the mammary gland structure in 3D culture. While Krause's study indicated that matrix composition greatly affected the morphology (11), another group has demonstrated that the standard 3D culture model (3D-base and embedded, Fig. 1D) did produce polarized mammary epithelial acini using normal mammary epithelial cells MCF-10A (12). In any event, with optimized ratio control between 4T1 and MEF, we were able to produce the unpolarized alveolar and ductal structures in the standard 3D culture by using 4T1 BCa cells and MEF fibroblasts. The fact that the complete/polarized alveolar/ductal mammary gland structure was not formed in our 3D co-culture may be consistent to the previous observation that the pathogenesis of epithelial tumor required the disruption of the intact polarized structure (12). With further modifications, the usage of such a 3D co-culture model can be widely applied in studying the interaction and interplay between breast tumor cells and stroma fibroblasts.

\section{ACKNOWLEDGEMENT}

This research project was supported by $\mathrm{NIH}$ grant CA107162 (YL). We thank Andrew Lu for reviewing the manuscript.

\section{CONFLICT OF INTEREST}

The authors have declared that no conflict of interest exists.

\section{REFERENCES}

1. Jemal A, Siegal R, Siegel R, Ward E, Xu J, Ward E. Cancer Statistics 2010. CA. A Cancer J Clin. 2010; 60: 277-300.

2. $\mathrm{Hu} \mathrm{M}$, Polyak K. Microenvironmental regulation of cancer development. Curr Opin Genet Dev. 2008; 18: 27-34.

3. Tlsty TD, Coussens LM. Tumor stroma and regulation of cancer development. Annu Rev Pathol. 2006; 1: 119-50.

4. Mueller MM, Fusenig NE. Friends or foes - bipolar effects of the tumor stroma in cancer. Nat Rev Cancer 2004; 4: 839-49.

5. Trimboli AJ, Cantemir-Stone ZC, Lu F, Wallace JA, et al. Pten in stromal fibroblasts suppresses mammary epithelial tumours. Nature 2009; 461: 1084-91.

6. Barcellos-Hoff $\mathrm{MH}$, Ravani SA. Irradiated mammary gland stroma promotes the expression of tumorigenic potential by unirradiated epithelial cells. Cancer Res. 2000; 60: 1254-60.

7. Maffini MV, Soto AM, Calabro JM, Ucci AA, Sonnenschein C. The stroma as a crucial target in rat mammary gland carcinogenesis. J Cell Sci. 2004; 117: 1287-90.

8. Maffini MV, Calabro JM, Soto AM, Sonnenschein C. Stromal regulation of neoplastic development: age-dependent normalization of neoplasic mammary cells by mammary stoma. Am J Pathol. 2005; 67: 1405-10.

9. Bhowmick NA, Neilson EG, Moses HL. Stromal fibroblasts in cancer initiation and progression. Nature 2004; 432: 332-7.

10. Kalluri R, Zeisberg M. Fibroblasts in cancer. Nat Rev Cancer 2006; 6: 392-401.

11. Krause S, Maffini MV, Soto AM, Sonneschein C. A novel 3D in vitro Culture model to study stromal-epithelial interactions in mammary gland. Tissue Engineer. 2008; 14: 261-71.

12. Debnath J, Muthuswamy SK, Brugge JS. Morphogenesis and oncogenesis of MCF-10A mammary epithelial acini grown in three-dimensional basement membrane culture. Methods 2003; 30: 256-68.

13. Littlepage LE, Egeblad M, Werb Z. Coevolution of cancer and stromal cellular responses. Cancer Cell 2005; 7: 499-500.

14. Yamada KM, Cukierman E. Modeling tissue morphogenesis and cancer in 3D. Cell 2007; 130: 601-10.

15. Ritter CA, Perez-Torres M, Rinehart C, Guix M, Dugger T, Engelman JA et al. Human breast cancer cells selected for resistance to trastuzumab in vivo overexpress epidermal growth factor receptor and ErbB ligands and remain dependent on the ErbB receptor network. Clin Cancer Res. 2007; 13: 4909-19.

16. Haycock JW. 3D cell culture: a review of current approaches and techniques. Methods Mol Biol. 2011; 695: 1-15.

17. Zhang J, Lu A, Li L, Yue J, Lu Y. p16 modulates VEGF expression via its interaction with HIF-1a in breast cancer cells. Cancer Invest. 2010; 28: 588-97.

18. Shaw KR, Wrobel CN, Brugge JS. Use of three-dimensional basement membrane cultures to model oncogene-induced changes in mammary epithelial morphogenesis. J Mammary Gland Biol Neoplasia 2004; 9: 297-310.

19. Lee GY, Kenny PA, Lee EH, Bissell MJ. Three-dimensional culture models of normal and malignant breast epithelial cells. Nat Methods 2007; 4: 359-65. 
20. Melillo G. Targeting hypoxia cell signaling for cancer therapy. Cancer Metastasis Rev. 2007;26: 341-52.

21. Liao D, Corle C, Seagroves TN, Johnson RS. Hypoxia-inducible factor-1a is a key regulator of metastasis in a transgenic model of cancer initiation and progression. Cancer Res. 2007; 67: 563-72.

22. Kalluri R. Basement membranes: structure, assembly and role in tumor angiogenesis. Nat Rev Cancer 2003; 3: 422-33.

23. Maniotis AJ, Folberg R, Hess A, Seftor EA, Gardner LM, Pe'er J, Trent JM, Meltzer PS, Hendrix MJ. Vascular channel formation by human melanoma cells in vivo and in vitro: vasculogenic mimicry. Ame J Pathol. 1999, 155: 739-52.

24. Feng $\mathrm{Y}$, Zhu $\mathrm{H}$, Ling T, et al. Effects of YC-1 targeting hypoxia-inducible factor 1 alpha in oesophageal squamous carcinoma cell line Eca109 cells. Cell Biol Int. 2011, 35: 491-7. 